\title{
Caprella ungulina Mayer, 1903 (Amphipoda: Caprellidae): epizoo de Paralomis granulosa (Hombron \& Jacquinot, 1846) (Decapoda: Lithodidae) en aguas de Magallanes, Chile
}

\author{
Caprella ungulina Mayer, 1903 (Amphipoda: Caprellidae): \\ epizoan of Paralomis granulosa (Hombron \& Jacquinot, 1846) \\ (Decapoda: Lithodidae) in Magellan waters, Chile
}

Álvaro Medina ${ }^{1}$, Tania Figueroa ${ }^{2} \&$ Juan I. Cañete ${ }^{2}$

\section{Resumen}

Se describe la epibiosis entre el anfípodo Caprella ungulina Mayer, 1903 y el crustáceo comestible Paralomis granulosa (centollón) capturados en aguas someras (50 a $100 \mathrm{~m}$ de profundidad) en cuatro sectores en los alrededores de la Isla Navarino $\left(55^{\circ} 03^{\prime} \mathrm{S}, 67^{\circ} 33^{\prime} \mathrm{O}\right)$ y en una localidad en la Isla Dawson (54 04' S, 70²4' O), Chile austral, durante la temporada de pesca de 2016. El material examinado ( $\mathrm{N}=289$ ejemplares de caprélidos) se recolectaron principalmente en los apéndices bucales (55\%) de P. granulosa. Se determinó que la epibiosis varió entre 1,8 y 14,8\% del número total de hospederos analizados, siendo frecuente el hallazgo de un individuo por centollón. La mortalidad de C. ungulina por efectos de la pesquería de $P$. granulosa efectuada el 2015 en Magallanes (1.965 ton; epibiosis promedio de $6,7 \%$ ) podría variar entre 255.450 y 1.061 .100 ejemplares. La mortalidad por pesca de $P$. granulosa podría llegar a afectar negativamente la dinámica poblacional de $C$. ungulina si se llega a demostrar una alta especificidad por este biohábitat y el uso de éste por individuos juveniles que no alcanzarían su maduración reproductiva. Se propone que la relación ecológica entre $C$. ungulina y $P$. granulosa podría ser definida como comensalismo por las siguientes causales: 1) el huésped es de mayor tamaño que la especie hospedada, 2) la relación relativamente específica entre ambas especies, 3) el posible uso de residuos alimentarios provistos por el hospedador, 4) un hábitat críptico, 5) el aporte de transporte y 6) abrigo físico.

\section{Palabras clave:}

Caprellidae, Caprella ungulina, epibiosis,

Paralomis granulosa, bentos marino

subantártico, mortalidad incidental por pesca, comensalismo.

\section{Abstract}

Caprella ungulina Mayer, 1903 is recorded from shallow waters (50 to $100 \mathrm{~m}$ depth) off Navarino Island $\left(55^{\circ} 03^{\prime} \mathrm{S}, 67^{\circ} 33^{\prime} \mathrm{W}\right)$ and Dawson Island (54 $04^{\prime} \mathrm{S}, 70^{\circ} 24^{\prime} \mathrm{W}$ ), austral Chile. The material examined ( $\mathrm{N}=289$ caprellids) was collected mainly in the mouthparts (55\%) of the edible crustacean Paralomis granulosa during 2016 fishery season. The percentage of epibiosis varied between 1.8 and $14.8 \%$ of the total host sampled, being collected mainly one caprellid by crab. The mortality of $C$. ungulina by effects of the $P$. granulosa fishery during 2015 in Magallanes (1,965 ton) produced between 255,450 and $1,061,100$ specimens (mean epibiosis of $6.7 \%$ $\pm 4,1 \%$ ). The mortality by fishing on $P$. granulosa could affect negatively the population dynamic of C. ungulina if is confirmed a high specificity for this biohabitat and the remotion of juveniles that could not reach the reproductive maturity. It is proposed that the ecological relationship between $C$. ungulina and $P$. granulosa could be defined as comensalism under

Instituto de Fomento Pesquero, Base Punta Arenas, Punta Arenas, Chile. alvaro.medina@ifop.cl

2 Dpto. Ciencias y Recursos Naturales, Facultad Ciencias, Universidad de Magallanes, Punta Arenas, Chile.

$\Sigma$ ivan.canete@umag.cl 
the following reasons: 1) the guest is largest than the species hosted, 2) the relatively specific relationship between both species, 3) use of food waste provided by the host, 4) use of host as a cryptic habitat, 5) the contribution in transport of the host and 6) physical shelter.

\section{Key words:}

Caprellidae, Caprella ungulina, epibiosis,

Paralomis granulosa, marine subantarctic bentos, incidental mortality for fishery, commensalism.

\section{INTRODUCCIÓN}

Caprella Lamarck, 1801 es el género de anfípodos de mayor biodiversidad en la familia Caprellidae con 162 especies en todo el mundo y está distribuido comúnmente en aguas poco profundas de zonas tropicales a templadas. Sin embargo, solamente 10 especies han sido recolectadas a profundidades superioresa 400 m(McCain\&Steinberg, 1970; Sittrop \& Serejo, 2006): C. equilibra Say (Schellenberg, 1926), C. horrida G. O. Sars (Mayer, 1890), C. microtuberculata G. O. Sars (Stephensen, 1944), C. punctata Boeck (Stephensen, 1944), C. rinki Stephensen (Stephensen, 1944), C. ungulina Mayer (Mayer, 1903; Vassilenko, 1974; Griffiths, 1977; Wicksten, 1982), C. gracilina Mayer (Vassilenko, 1974), C. bathyalis Vassilenko (Vassilenko, 1972, 1974), C. pacifica Vassilenko (Vassilenko, 1972, 1974) y C. longicirrata Vassilenko (Vassilenko, 1974). De estas especies, C. ungulina es la única especie que ha sido descrita como epibionte que vive asociado a crustáceos litódidos (Griffiths, 1977; Wicksten, 1982). C. ungulina tienen una amplia distribución geográfica, habiendo sido colectada en la zona batial frente a Japón, siendo capturada también en las partes bucales de crustáceos litódidos (Takeushi et al. 1989) y en aguas profundas $(1.067 \mathrm{~m})$ frente a la costa de Brasil sobre el litódido Paralomis formosa (Sittrop \& Serejo, 2006).

En el presente estudio se describe cuantitativamente la epibiosis que realiza el anfípodo caprélido Caprella ungulina sobre las partes bucales y otras zonas corporales del crustáceo litódido de interés comercial Paralomis granulosa capturados en las proximidades de la Isla Navarino, Canal Beagle y en un sector adyacente de la Isla Dawson, Chile, durante la temporada de pesca 2016 y se aportan evidencias bibliográficas para definir el tipo de relación ecológica existente entre ambas especies de crustáceos. El hallazgo del anfípodo $C$. ungulina en poblaciones de $P$. granulosa es atípico debido a que la pesca de este crustáceo se realiza en aguas de menos de $100 \mathrm{~m}$ de profundidad, porque C. ungulina se ha citado para zonas profundas de los océanos Pacífico y Atlántico, y porque los registros previos en la costa de Magallanes han sido referidos principalmente a la descripción original, careciéndose de antecedentes cuantitativos de este tipo de epibiosis (De Broyer \& Rauschert, 1999; Thiel et al. 2003; Vinuesa et al. 2015).

El objetivo de esta investigación es demostrar cuantitativamenteel gradodeepibiosisque muestraesta especie de caprélido sobre el crustáceo de importancia comercial P. granulosa, aportar antecedentes para identificar el tipo de relación ecológica existente entre ambos crustáceos, corroborar la existencia de ojos en ejemplares de zonas someras y estimar el impacto de la pesca de $P$. granulosa en la mortalidad incidental de $C$. ungulina. Preliminarmente existe evidencia de la pesencia de $C$. ungulina como epibionte sobre Neolithodes asperrimus Barnard 1947, Paralomis multispina Benedict, 1895, Lithodes aequispina Benedict, 1895 y Paralomis formosa Henderson, 1888 (Griffiths, 1977; Takeuchi et al. 1989; Sittrop \& Serejo, 2006) y sobre P. granulosa en la costa patagónica de Argentina (Vinuesa et al. 2015). Sin embargo, no se ha cuantificado este tipo de epibiosis ni se ha determinado el rol y el tipo de relación ecológica de esta interacción. Estudios recientes sobre las comunidades epibióticas asociadas con el caparazón de litódidos muestra que existe una gran biodiversidad de incrustaciones biológicas que recién comienzan a ser estudiadas en forma cuantitativa (Dvoretsky \& Dvoretsky, 2008, 2009, 2010, 2013; Fernandez-Leborans, 2010). Las razones que justifican este tipo de estudios se relacionan con el impacto de la pesquería de grandes crustáceos sobre la biodiversidad de la fauna asociada, potenciales enfermedades parasitarias o plagas que podrían ser introducidas a otros países e influencia del cambio climático sobre las comunidades bióticas. Un aspecto novedoso que incorpora el presente estudio es el impacto negativo que podría traer asociada la mortalidad incidental por pesca del hospedador sobre la especie huésped ejemplificado en dos especies de crustáceos subantárticos. 


\section{MATERIALES Y MÉTODOS}

\section{Captura de P. granulosa}

Los ejemplares de $P$. granulosa fueron capturados mediante trampas localizadas en cinco áreas en el sector este y sur-oeste de la Isla

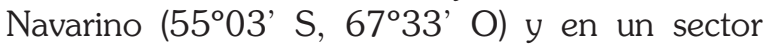
adyacente a la Isla Dawson (5404' S, 70²4' O), zona austral de Chile, a profundidades entre 50 y 100 m durante la temporada de pesca 2016, entre los meses de agosto a noviembre (Tabla 1). Las trampas permanecieron entre 24 y 48 horas asentadas sobre el fondo antes de ser izadas a la superficie. La carnada utilizada para atraer a $P$. granulosa consistió mayormente de cabeza de merluza (Merluccius australis) congelada.

Todos los ejemplares de $P$. granulosa analizados correspondieron a machos (longitud de cefalotórax, LC, mayor a $80 \mathrm{~mm}$, que corresponde a la talla mínima legal de captura) los que fueron desembarcados en el muelle de Caleta Barranco Amarillo, Punta Arenas, al menos 5 a 6 días después de la captura. Los ejemplares de $P$. granulosa fueron transportados desde la zona de pesca hacia Punta Arenas en embarcaciones de acarreo, las cuales en ciertas ocasiones también transportaban en conjunto a la especie Lithodes santolla (centolla).

Determinación de epibiosis y

localización de C. ungulina en $P$. granulosa

De la muestra total de $P$. granulosa desembarcada (144.580 kg) se extrajo una muestra de ejemplares (3.072 ind.) para verificar la existencia de
C. ungulina en cada uno de ellos. Una vez identificada la presencia de $C$. ungulina, se procedió a contabilizar el número de ejemplares epizoos, determinando su posición en el cuerpo del centollón. El porcentaje de epibiosis fue estimado contabilizando el número de centollones con presencia del anfípodo, además de determinar el número de anfípodos presentes en cada ejemplar (Tabla 1). Estos índices son equivalentes al porcentaje de prevalencia (proporción de P. granulosa con epibiosis) y la intensidad (número de C. ungulina presente en cada ejemplar de centollón) (Bush et al. 1997; Dvoretsky \& Dvoretsky, 2013).

\section{Identificación taxonómica de C. ungulina}

La identificación taxonómica de C. ungulina fue realizada tomando en consideración las siguientes descripciones: Mayer (1903), Schellenberg (1931), Griffiths (1977), Takeuchi et al. (1989) y Sittrop y Serejo (2006). Otras fuentes de información comparativa se basaron en las descripciones de otros caprélidos descritos para la costa de Chile (Guerra-García \& Thiel, 2001) así como el registro previo de su presencia en aguas de Magallanes (De Broyer \& Rauschert, 1999; De Broyer et al. 2004; Thiel et al. 2003) (Fig. 1). Cada ejemplar fue observado bajo una lupa estereoscópica para corroborar la existencia de ojos, aspecto que está en permanente discusión en relación a la ausencia de ojos en ejemplares recolectados en zonas del mar profundo.

Todos los ejemplares fueron fijados en etanol absoluto y fueron depositados en la Sala Sistemática y de Colecciones de Referencia del Instituto de la Patagonia, Universidad de Magallanes ( $\mathrm{N}^{\circ}$ Ingreso:

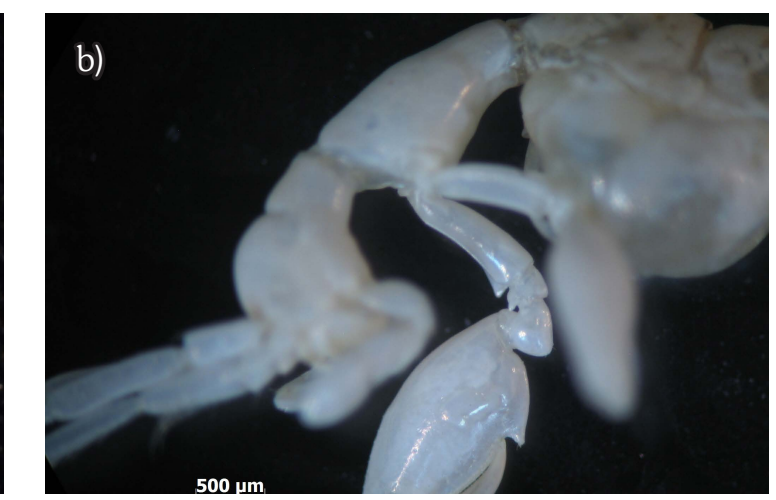

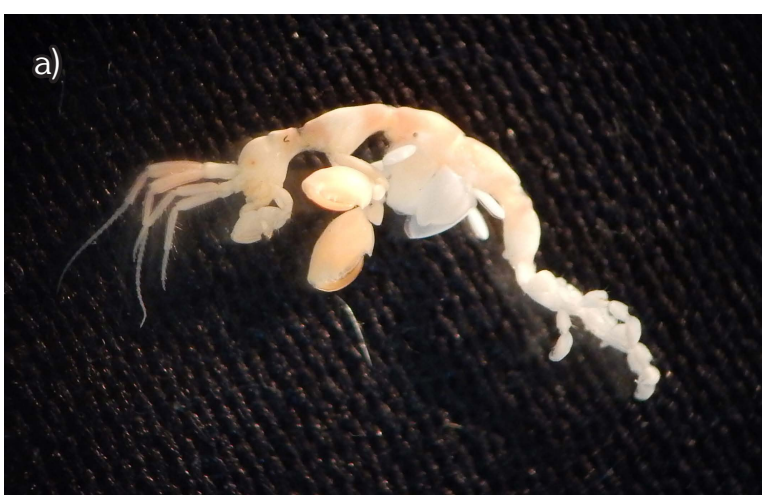

Fig. 1. a) Vista lateral de un ejemplar adulto de Caprella ungulina recolectado como epizoo del crustáceo Paralomis granulosa en los alrededores de Isla Navarino, Chile, b) Detalle del gnatopodo 2 de Caprella ungulina. 
A. MEDINA et al.

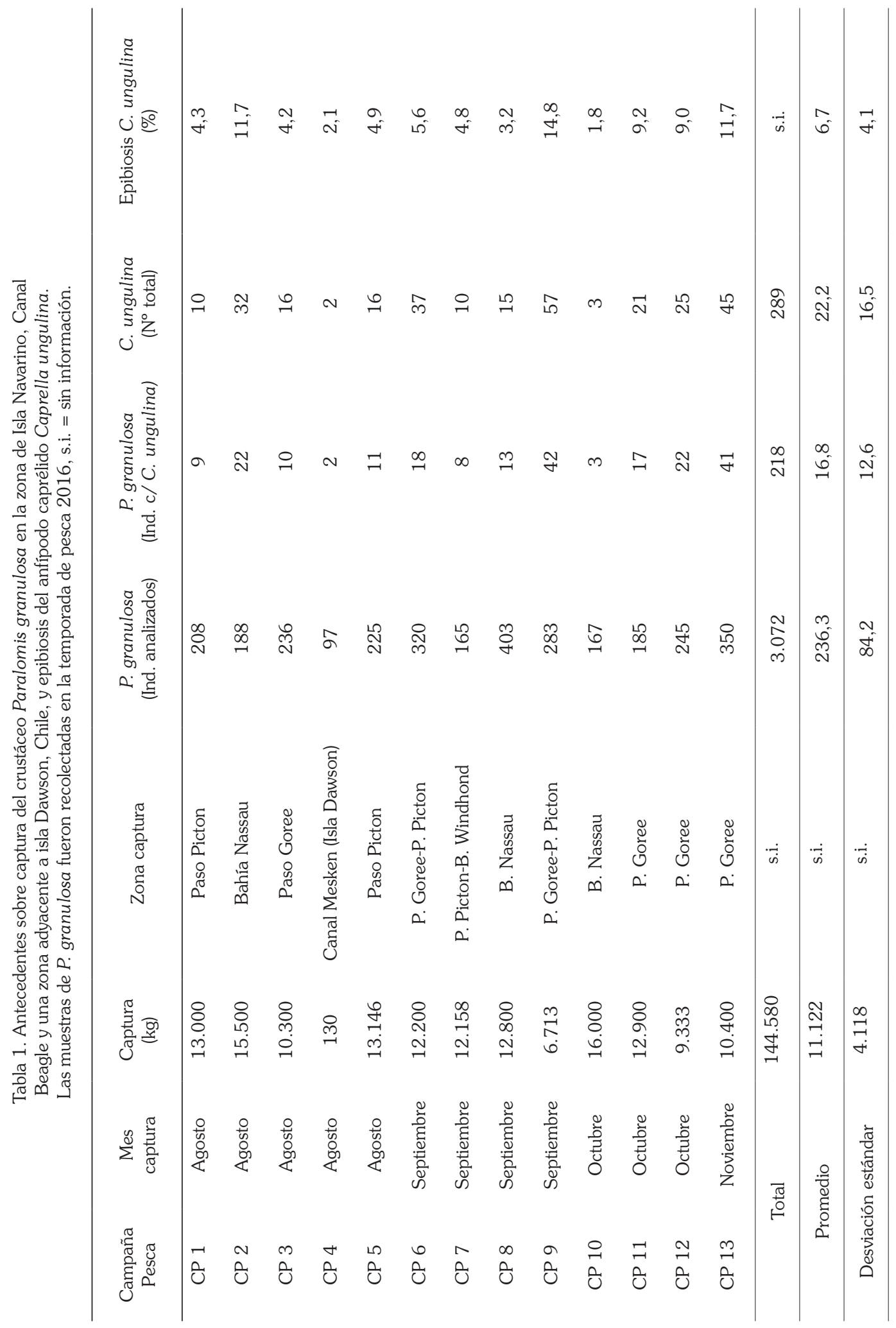


2898-5-16-625; $\quad \mathrm{N}^{\circ} \quad$ Catálogo: UMAG-CIIPART-700595).

Estimación de mortalidad incidental de C. ungulina

Para estimar el impacto de la pesca del litódido $P$. granulosa en la captura incidental de C. ungulina se analizó la relación longitud total (LT) us peso total (PT) calculada para el mes de abril de 2014 para los desembarques realizados en Puerto Williams dentro del programa de seguimiento de pesquerías de crustáceos bentónicos desarrollado por el Instituto de Fomento Pesquero (IFOP) (Daza \& Almonacid, 2015).

Esta función de regresión generada para P. granulosa $\left(\mathrm{R}^{2}=0,93 ; \mathrm{N}=3.483\right.$ ejemplares; $\mathrm{PT}=0,0003 * \mathrm{LT}^{3,15}$ ) permitió estimar la cantidad de ejemplares machos adultos mayores a $80 \mathrm{~mm}$ de LC que podrían haber sido capturados de acuerdo al desembarque total realizado el año 2015 para la región de Magallanes (1.965 toneladas) (Sernapesca, 2015).

Basado en esta función de regresión, un centollón de $90 \mathrm{~mm}$ de LC tiene un peso total aproximado de $500 \mathrm{~g}$ (Fig. 64 de Daza \& Almonacid, 2015) y una tonelada de $P$. granulosa equivaldría a 5.000 ejemplares de $90 \mathrm{~mm}$ LC. Por lo tanto, se estimó que durante la temporada de pesca se capturaron aproximadamente 9.825.000 ejemplares en la Región de Magallanes. Este valor fue multiplicado por

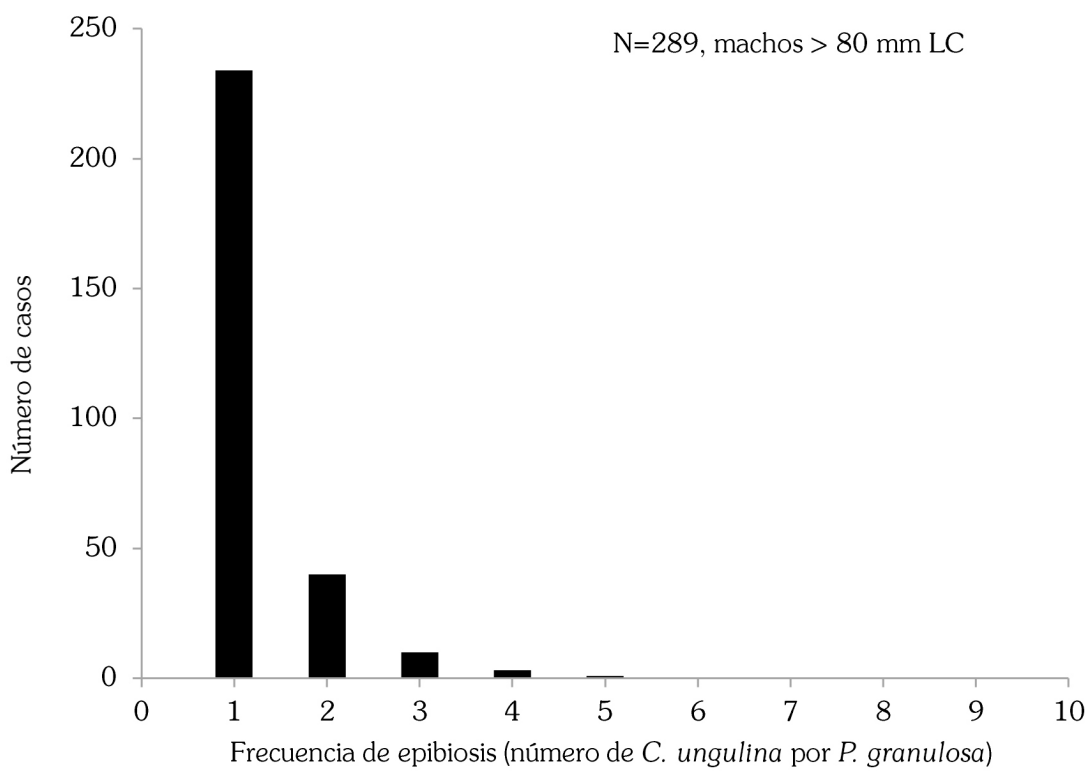

Fig. 2. Frecuencia de epibiosis de Caprella ungulina sobre ejemplares machos adultos del crustáceo Paralomis granulosa capturados en los alrededores de Isla Navarino y una localidad en isla Dawson, Chile. el porcentaje de epibiosis de C. ungulina estimado, cuyo rango varió entre 1,8 y $14,8 \%$ de la muestra de $P$. granulosa, considerando solamente el valor promedio y su desviación estándar (6,7\%; Tabla 1), el cual se utilizó para estimar la mortalidad incidental de anfípodos caprélidos asociada a la mortalidad por pesca de P. granulosa.

\section{RESULTADOS}

Abundancia, prevalencia e intensidad de C. ungulina en $P$. granulosa

De aproximadamente $145.000 \quad \mathrm{~kg}$ desembarcados de $P$. granulosa proveniente de 6 embarcaciones distintas que trabajaron alrededor de la isla Navarino y en el Canal Mesken (Isla Dawson) en la temporada de pesca de 2016 (Tabla 1), se extrajeron 3.072 ejemplares para analizar la presencia de $C$. ungulina y el porcentaje de epibiosis. De este total, solamente 218 centollones presentaron este anfípodo (porcentaje promedio de 6,7 $\pm 4,1$ $\%)$, recolectándose un total de 289 ejemplares de C. ungulina (Tabla 1). Se observó que el rango más recurrente de C. ungulina en cada ejemplar de $P$. granulosa fue de un anfípodo por centollón adulto (81,3\%). Sin embargo, se contabilizaron hasta 5 anfípodos por centollón analizado (1 caso, $0,35 \%$, Fig. 2).

\section{$\mathrm{N}=289$, machos $>80 \mathrm{~mm} \mathrm{LC}$}


Localización de C. ungulina en el cuerpo de P. granulosa

Todos los ejemplares de C. ungulina fueron recolectados en el extremo anterior de P. granulosa. El mayor porcentaje se capturó en los apéndices bucales (55\%; Fig. 3a) y en la base de las antenas (35\%; Fig. 3b), mientras que un menor porcentaje fue capturado en el margen anteroventral del cefalotórax (10\%; Fig. 3c). Lo más llamativo es el grado de mimetismo que experimenta $C$. ungulina respecto a la coloración del extremo anterior de $P$. granulosa.

Presencia de ojos en C. ungulina

Todos los ejemplares analizados presentaron ojos visibles al ser observados bajo la lupa estereoscópica.

Mortalidad incidental de C. ungulina

Asumiendo que el desembarque total de P. granulosa para el año 2015 corresponde aproximadamente a la extracción total de 9.825.000 ejemplares adultos y considerando que el porcentaje de epibiosis promedio es de 6,7 \pm $4,1 \%$ (Tabla 1 ), implicaría que en cada temporada de pesca pueden morir incidentalmente entre 255.450 y 1.061 .100 ejemplares de C. ungulina asociado a la mortalidad por pesca de $P$. granulosa. Al considerar el rango de variación del porcentaje de epibiosis (1,8 y 14,8\%; Tabla 1), el rango de mortalidad incidental de $C$. ungulina varía entre 98.250 y 1.454 .100 individuos.

\section{DISCUSIÓN}

En el presente estudio se aporta evidencia cuantitativa de la epibiosis del anfípodo caprélido Caprella ungulina sobre ejemplares machos del crustáceo litódido Paralomis granulosa en la zona austral de Chile, y además asigna tentativamente el tipo de relación ecológica existente entre ambas especies. Los registros previos se basaban en la captura de algunos ejemplares de litódidos recolectados ocasionalmente y en baja abundancia en zonas del mar profundo. De esta manera, $C$. ungulina posee un amplio rango de distribución
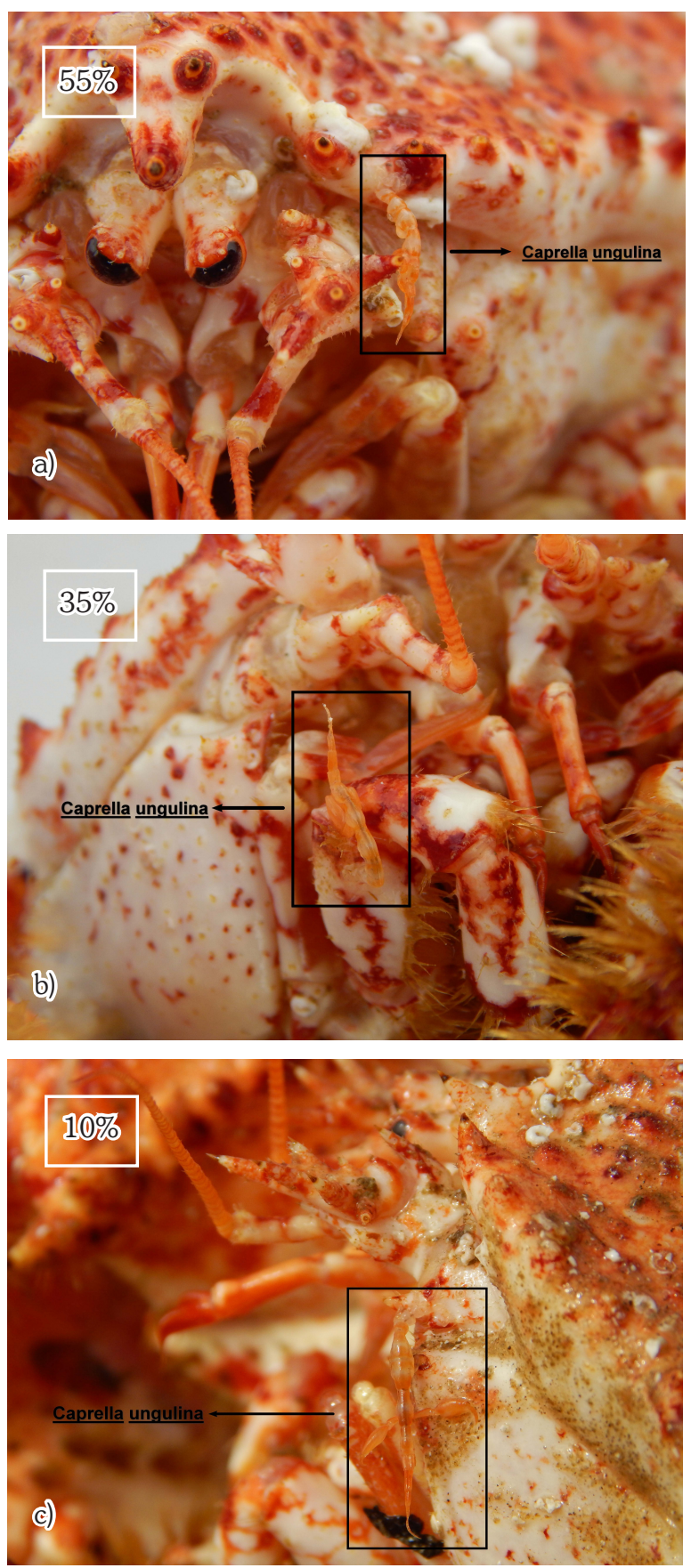

Fig. 3. Epibiosis de Caprella ungulina sobre el extremo anterior y zona bucal de ejemplares adultos del crustáceo Paralomis grabnulosa capturados en los alrededores de Isla Navarino, Chile: a) zona bucal, b) zona basal de la Antena 1, c) margen anterolateral del extremo anterior. En los recuadros se indica la frecuencia de ocurrencia de C. ungulina en cada zona. 
batimétrica en el Océano Pacífico y en el Océano Atlántico, habitando aguas de baja temperatura $\left(<7^{\circ} \mathrm{C}\right)$ (McCain \& Steinberg, 1970; Sittrop \& Serejo, 2006).

¿Presencia de ojos en C. ungulina en aguas magallánicas?

Mediante el presente estudio se demuestra que los ejemplares de C. ungulina de áreas someras si poseen ojos y que todos los ejemplares recolectados como epizoos de $P$. granulosa poseen ojos (Fig. 1a). Según la descripción original de Mayer (1903), C. ungulina tiene ojos. Griffiths (1977) no mencionó los ojos en su descripción. Takeuchi et al. (1989), quienes observaron ejemplares de zonas profundas recolectados frente a la costa de Columbia Británica, afirmó que ni los machos ni las hembras tenían ojos. Una explicación ante la ausencia de ojos en $C$. ungulina recolectados en zonas profundas puede ser la alta presión y la gran oscuridad existente en este ambiente.

Thurston \& Bett (1993) afirmaron que en variadas especies de anfípodos que habitan en áreas costeras someras presentan ejemplares con ojos visibles, mientras que aquellas poblaciones de zonas profundas carecen de ojos visibles. Estos autores aportaron antecedentes en algunos géneros de anfípodos gamáridos tales como Ampelisca Kroyer, 1842; Byblis Boeck, 1871; Argissa Boeck, 1871; Listriella Barnard, 1959; Schisturella Norman, 1900 y Heterophoxus Shoemaker, 1925.

\section{Prevalencia de C. ungulina en $P$. granulosa}

El porcentaje de epibiosis determinado en el presente estudio es bajo dado que menos del $15 \%$ de los ejemplares de P. granulosa analizados mostró epibiosis por parte de C. ungulina (Tabla 1), tomando en consideración el tamaño muestreal y que en otras especies de litódidos introducidos al Mar de Barents (Paralithodes camtschaticus), algunos anfípodos invasores (género Ischyrocerus) muestran niveles de prevalencia sobre el $40 \%$ (Dvoretsky \& Dvoretsky, 2010) y en especies de copépodos harpacticoídeos comensales muestran valores superiores al $20 \%$ de prevalencia en el sistema branquial (Dvoretsky \& Dvoretsky, 2013).
Dada la captura de sólo ejemplares machos no se puede predecir qué niveles de epibiosis podrían ser comunes en ejemplares de $P$. granulosa de sexo femenino. Sin embargo, Dvoretsky y Dvoretsky (2010) han demostrado que no existen diferencias estadísticas en la composición y biodiversidad de la epifauna asociada al caparazón de $P$. camtschaticus de diferente sexo, pero sí parece afectar la edad. De este modo, ejemplares de mayor tamaño muestran mayor prevalencia y biodiversidad de epizoos que ejemplares jóvenes o con mayor recambio del caparazón.

Otro aspecto llamativo es la ausencia de C. ungulina sobre los ejemplares de L. santolla observados para efectos comparativos a pesar de provenir de las mismas zonas de pesca alrededor de la isla Navarino, aunque de áreas más someras ( $<50$ m profundidad) y de existir evidencias de que este anfípodo ha sido recolectado previamente en otros miembros del género, tal como en Lithodes aequispina (Griffiths, 1977; Takeuchi et al. 1989). Dvoretsky y Dvoretsky (2010) describen que algunas especies de anfípodos comensales epizoos de $P$. camtschaticus muestran mayor porcentaje de prevalencia ( $>55 \%$ ) en zonas profundas (120 a $180 \mathrm{~m}$ ) que zonas de menor profundidad (5 a 40 $\mathrm{m})$.

En nuestro caso, se revisaron alrededor de 600 ejemplares de talla comercial de L. santolla (centolla; mayores a 120 mm de LC), observándose los apéndices, antenas, zona bucal o espinas para detectar la presencia de C. ungulina (A. Medina, Obs. Pers.). Sin embargo, C. ungulina no fue observado en ningún ejemplar de centolla macho. De esta manera, habrá que investigar con mayor detalle el por qué no existe epibiosis de C. ungulina sobre ejemplares adultos de Lithodes santolla. Adicionalmente, esta evidencia puede demostrar algún grado de especificidad entre $C$. ungulina y $P$. granulosa en la zona austral de Chile.

Intensidad de epibiosis de

C. ungulina en $P$. granulosa

Interesante resulta del análisis de esta relación epibiótica entre ambos crustáceos es que en la mayoría de los casos existe solamente un caprélido por centollón (Fig. 2). Esto coincide con los hallazgos previos efectuados para representantes del género 
Paralomis donde se ha detectado sólo una Caprella por individuo (e.g. P. multispina; Takeushi et al. 1989). Este grado de epibiosis podría ser afectado por el transporte, manipulación e interacción entre centollones durante su traslado a los puertos de desembarque en Porvenir o Punta Arenas, localizados casi a $500 \mathrm{~km}$ de la zona de pesca.

Localización de C. ungulina en el caparazón de $P$. granulosa

C. ungulina habita en diferentes zonas del extremo anterior de $P$. granulosa, aunque muestra mayor prevalencia en la zona bucal (Figs. 3a-c). Investigaciones sobre la comunidad epibiótica asociada a diferentes zonas corporales del litódido $P$. camtschaticus han demostrado que 8 de 9 especies de macroinvertebrados pertenecientes al menos a 6 fila muestran preferencia por la parte superior del caparazón (18 a 78\%), mientras que una especie de anfípodo comensal selecciona preferentemente las branquias, la zona bucal y los apéndices ambulacrales (Dvoretsky \& Dvoretsky, 2010), donde se ha detectado además un cambio ontogenético en las áreas utilizadas. Por ejemplo, los adultos se localizan preferentemente en la zona bucal, mientras que los ejemplares juveniles se localizan principalmente en las branquias. En nuestro caso, no se observó dicho comportamiento poblacional.

Todos los ejemplares de C. ungulina recolectados sobre $P$. granulosa representan estadios juveniles. Una evidencia la aporta la longitud del pereonito 1 de los ejemplares de $C$. ungulina recolectados sobre ejemplares adultos de P. granulosa en la zona austral de Chile (Fig. 1a), los cuales muestran escaso desarrollo en relación al ejemplar de la zona batial de Japón esquematizado por Takeushi et al. (1989), donde se observa muy prolongado. Esto podría sugerir que los ejemplares analizados en el presente estudio podrían ser juveniles o machos sexualmente premaduros y por lo tanto tal vez esta relación simbiótica podría ser catalogada como facultativa ya que no hay un uso permanente del biosustrato. Nuestros ejemplares mostraron un pereonito 1 de longitud 2,2 $\pm 0,5$ mm y un gnatopodo 2 de 1,6 $\pm 0,3$ mm de longitud, mientras que el ejemplar macho de Takeushi et al. (1989) posee una longitud de $6 \mathrm{~mm}$ y 3,5 mm, respectivamente. Adicionalmente, la morfología corporal de los ejemplares de zonas profundas puede presentar un fenotipo distinto respecto de ejemplares co-específicos habitando zonas someras de escasa profundidad.

Una de las posibles razones para que $C$. ungulina habite en esta zona del caparazón de $P$. granulosa se podría deber a que sobre el cefalotórax, apéndices y bajo el abdomen existe una fuerte epibiosis de una especie no identificada de poliqueto perteneciente a la familia Spirorbidae que es muy recurrente en ejemplares adultos y puede afectar al $100 \%$ de los ejemplares capturados en la zona de Isla Navarino, Chile (Fig. 4). También se puede postular que $C$. ungulina sea una especie suspensivivora y aproveche la corriente o flujo de agua generado al caminar el hospedero y de alimentarse del material particulado retenidos en las setas de los apéndices bucales.

Desde el punto de vista trófico, los anfípodos caprélidos se alimentan como suspensivoros (Caine, 1979), mientras que otras especies ramonean desarrollando un raspaje sobre el substrato (Takeuchi \& Hirano, 1995). También podrían aprovechar las fecas producidas por los poliquetos Spirorbidae adheridos en la parte superior del cefalotórax.

Adicionalmente, para algunos caprélidos se han descrito hábitos predadores (Caine, 1980). Los caprélidos también constituyen una fuente de alimento importante para peces costeros (Caine, 1987, 1989, 1991), los cuales podrían ejercer un fuerte consumo, regulando la densidad poblacional (Caine, 1991). De esta manera, C. ungulina podría aprovechar este tipo de relación ecológica para lograr transporte, alimento y de acuerdo al patrón de coloración relativamente similar al color del caparazón de P. granulosa (Fig. 3a-c), éste podría actuar como un sustrato para lograr mimetizarse y evitar la depredación.

Cabe destacar que si bien los anfípodos caprélidos se encuentran adaptados a vivir en el bentos, algunas especies viven como epibiontes en algas y fanerógamas marinas o asociados a hidrozoos, briozoos y también a esponjas (McCain, 1968; Laubitz, 1970; Laubitz \& Mills, 1972; Caine, 1998; Guerra-García, 2001). Algunas especies establecen asociaciones más específicas con otros invertebrados marinos, como corales gorgónidos (Lewbel, 1978), 


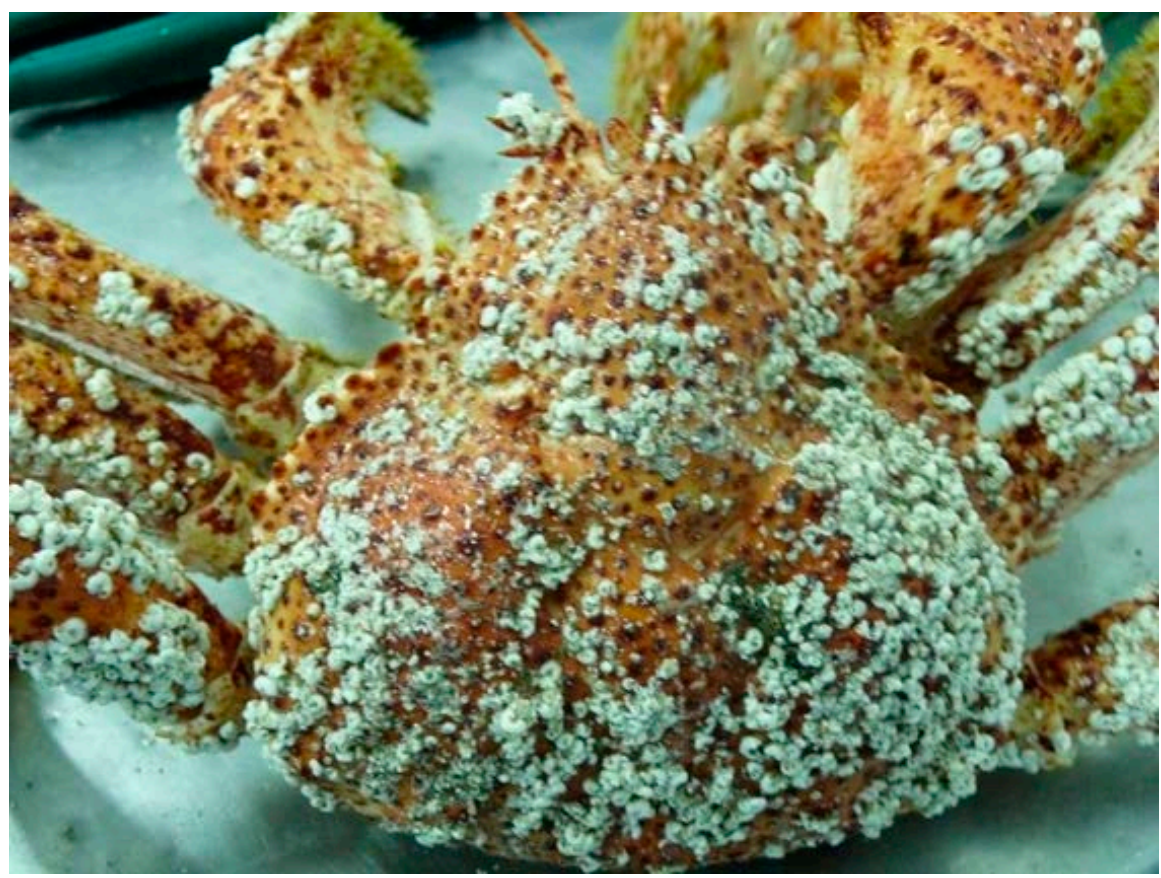

Fig. 4. Epibiosis del cefalotórax de ejemplares adultos machos del crustáceo Paralomis granulosa por parte de un poliqueto Spirorbidae no identificado, Isla Navarino, zona austral de Chile.

grandes crustáceos (Martin \& Pettit, 1998) y equinodermos (Vader, 1978).

¿Comensalismo entre

C. ungulina y $P$. granulosa?

La relación ecológica existente entre $C$. ungulina y $P$. granulosa podría ser definida como una simbiosis de tipo comensal. Las principales razones para asignar este tipo de relación ecológica son: 1) el huésped es de mayor tamaño que la especie hospedada, 2) la relación relativamente específica entre ambas especies, 3) el posible uso de residuos alimentarios provistos por el hospedador, 4) un hábitat críptico, 5) el transporte para la especie hospedada y 6) abrigo físico (Cheng, 1967; Rozbaczylo \& Cañete, 1993; Martin \& Britayev, 1998). Sin embargo, restarían otros análisis futuros para demostrar la existencia de este tipo de relación ecológica entre ambas especies de crustáceos tales como: nuevos hallazgos de este tipo de relación entre miembros congenéricos de estos crustáceos, mayor especificidad, análisis de variabilidad espacial en los niveles $e$ intensidad de la simbiosis y adaptaciones morfológicas, conductuales, fisiológicas y reproductivas entre ambas especies (Martin \& Britayev, 1998).

Las relaciones ecológicas entre anfípodos y otras especies de crustáceos pueden ser divididas en cinco categorías (Vader \& Tandberg, 2015): 1) anfípodos que habitan en la epifauna adherida al caparazón de grandes crustáceos, 2) anfípodos que viven en relación directa con la superficie de la especie hospedadora, 3) anfípodos que viven principalmente al interior de la masa ovígera de los huéspedes, 4) anfípodos que habitan en túneles o madrigueras permanentes de crustáceos infaúnicos y 5) anfípodos que pueden habitar al interior o sobre las conchas de gastéropodos ocupadas por cangrejos ermitaños o miembros de la familia Paguridae. De esta manera, la relación establecida entre C. ungulina y $P$. granulosa se ajusta a la categoría 2, donde el anfípodo habita en las antenas, partes bucales y zona antero-ventral del extremo anterior del caparazón del centollón.

Muchos de los anfípodos pertenecientes a esta categoría se caracterizan por poseer pereiópodos prehensiles para adherirse al huésped (Vader \& Tandberg, 2015). Adicionalmente, muchos de estos anfípodos parecen ser simbiontes 
que viven en forma específica sobre determinados hospedadores, siendo la excepción algunas especies de anfípodos de amplia distribución geográfica tal como C. ungulina Mayer, 1903 y C. bathytatos Martin \& Pettit, 1998, las cuales han sido recolectadas en una amplia variedad de especies de litódidos (Vader \& Tandberg, 2015).

Mortalidad incidental de C. ungulina por efectos de la pesca de P. granulosa

El grado de epibiosis reflejado en la Fig. 2 podría ser un estimador del grado de mortalidad incidental que genera la pesquería de $P$. granulosa sobre el anfípodo caprélido $C$. ungulina. P. granulosa es una especie de importancia comercial en Chile y Argentina, y sólo en el año 2015 se extrajeron 1.965 toneladas en la región de Magallanes, Chile (Sernapesca, 2015). Proyectando los resultados obtenidos y que se pueden observar hasta 5 caprélidos por centollón (Fig. 2), los niveles de mortalidad provocados por la pesquería de este litódido podrían afectar negativamente la dinámica poblacional de este anfípodo si se llega a demostrar una alta especificidad entre ambas especies, una relación simbiótica y si se corrobora que el estrato poblacional que vive asociado a $P$. granulosa solo corresponde a ejemplares juveniles. En este último caso, los ejemplares no alcanzarían a madurar sexualmente, afectando por lo tanto al éxito reproductivo de esta especie de caprélido. Ninguno de los ejemplares de C. ungulina observados portaban huevos.

Por lo tanto, la pesquería de $P$. granulosa podría no afectar directamente al esfuerzo reproductivo de C. ungulina. Sin embargo, si se llegara a sobrexplotar las poblaciones de P. granulosa en la zona austral de Chile podría representar un impacto negativo sobre la biodiversidad de este anfípodo caprélido austral ya que se estaría eliminando el hábitat en que ellos viven. Además, esta situación plantea la interrogante acerca del tipo de hábitat que podrían utilizar las poblaciones de ejemplares adultos de $C$. ungulina o si pueden utilizar otro tipo de biohábitats en la zona de estudio.

\section{CONCLUSIÓN}

El presente estudio desea llamar la atención acerca del potencial impacto que podría ocasionar la invasión del continente antártico por parte de crustáceos litódidos subantárticos (Smith et al. 2011; Griffiths et al. 2013) y su correspondiente biodiversidad de epizoos comensales y especies parasitarias. Hasta el presente, al menos 4 especies de ecto y endoparásitos han sido reportados en $P$. granulosa y $L$. santolla en la zona austral de Sud América (los isópodos Liriopsis pygmaea y Pseudione tuberculata, el cirripedio rizocéfalo Briarosaccus callosus y una especie de microsporidia recientemente descrito a nivel genérico y específico (Lovrich \& Tapella, 2014; Stevens, 2014; Stentiford et al. 2014; Cañete et al. 2017). El seguimiento permanente de este tipo de relaciones ecológicas es importante y quizás un requerimiento obligatorio para incorporar en futuros planes de manejo de ambos recursos subantárticos.

Cabe destacar que el material de C. ungulina recolectado ha sido fijado en etanol absoluto para futuros análisis secuenciación de ADN y otros aspectos moleculares que sean de interés de comparar con ejemplares del mar profundo o de otras zonas someras frías del Océano Pacífico y Atlántico.

\section{AGRADECIMIENTOS}

Se agradece la colaboración y autorización prestada por el Biólogo Marino Erik Daza (Instituto de Fomento Pesquero, Base Punta Arenas), por dar la oportunidad de hacer uso de las muestras de crustáceos litódidos de los desembarques 2016 y permitir la divulgación de estos resultados para fines de esta publicación e igualmente agradecer la ayuda y los consejos de la observadora científica del IFOP Alejandra Valdebenito, especialmente en los muestreos realizados en la caleta. Se extienden los agradecimientos a los revisores de este manuscrito, quienes han mejorado el contenido de éste.

\section{LITERATURA CITADA}

Aoki, M. (1988). Factors affecting population fluctuations of caprellid amphipods inhabiting Sargassum patens bed (preliminary report). Benthos Research, 32, 42-49. 
Bush, A. O., Lafferty, K. D., Lotz, J. M., \& Shostak, A. W. (1997). Parasitology Meets Ecology On Its Own Terms: Margolis et al. Revisited. The Journal of Parasitology, 83, 575-583.

Caine, E. A. (1979). Functions of swimming setae within caprellid amphipods (Crustacea). Biological Bulletin, 156, 169-178.

Caine, E. A. (1980). Ecology of two littoral species of caprellid amphipods (Crustacea) from Washington, USA. Marine Biology, 56, 327-335.

Caine, E. A. (1987). Potential effect of floating dock communities on a South Carolina estuary. Journal of Experimental Marine Biology and Ecology, 108, 83- 91.

Caine, E. A. (1989). Caprellid amphipod behavior and predatory strikes by fish. Journal of Experimental Marine Biology and Ecology, 126, 173-180.

Caine, E. A. (1991). Caprellid amphipods: fast food for the reproductively active. Journal of Experimental Marine Biology and Ecology, 148, 27-33.

Caine, E. A. (1998). First case of caprellid amphipod-hydrozoan mutualism. Journal of Crustacean Biology, 18, 317-320.

Cañete, J. I., Díaz-Ochoa, J. A., Figueroa, T., \& Medina, A. (2017). Infestation of Pseudione tuberculata (Isopoda: Bopyridae) on juveniles of Lithodes santolla (Magellan region, Chile): a spatial mesoscale analysis. Latin American Journal of Aquatic Research, 45, 79-93.

Cheng, T. C. (1967). Marine molluscs as host for symbiosis. En F. S. Russell (Ed.), Advances in Marine Biology 5 (424 p.). London: Academic Press.

Daza, E., \& Almonacid, E. (2015). Programa de Seguimiento Pesquerías, Crustáceos Bentónicos, 2014 - Recurso Centolla y Centollón, XII Región, 2014. Convenios de Desempeño 2014, Subsecretaría de Economía, Chile, Informe Final, Instituto de Fomento Pesquero, Punta Arenas, 221 pp. + anexos.

De Broyer, C., \& Rauschert, M. (1999). Faunal diversity of the benthic amphipods (Crustacea) of the Magellan region as compared to the Antarctic (preliminary results). Scientia Marina, 63, 281-293.

De Broyer, C., Guerra-García, J. M., Takeuchi, I., Robert, H., \& Meerhaeghe, A. (2004). Biodiversity of the Southern Ocean: a catalogue of the Antarctic and sub-Antarctic Caprellidae and Cyamidae (Crustacea: Amphipoda) with distribution and ecological data. Bulletin de l'Institut Royal des Sciences Naturelles de Belqique, Sciences de la Terre . Biologie, 74, 61-99.

Dvoretsky, A. G., \& Dvoretsky, V. G. (2008). Epifauna associated with the northern stone crab Lithodes maia in the Barents Sea. Polar Biology, 31, 1149-1152.

Dvoretsky, A. G., \& Dvoretsky, V. G. (2009). Fouling community of the red king crab Paralithodes camtschaticus (Tilesius 1815), in a subarctic fjord of the Barents Sea. Polar Biology, 32, 1047-1054.

Dvoretsky, A. G., \& Dvoretsky, V. G. (2010). Epifauna associated with an introduced crab in the Barents Sea: a 5-year study. ICES Journal of the Marine Sciences, 67, 204214.

Dvoretsky, A. G., \& Dvoretsky, V. G. (2013). Copepods associated with the red king crab Paralithodes camtschaticus (Tilesius, 1815) in the Barents Sea. Zoological Studies, 52, 17-23.

Fernandez-Leborans, G. (2010). Epibiosis in Crustacea: An Overview. Crustaceana, 83(5), 549-640.

Griffiths, C. L. (1977). Deep sea amphipods from west of Cape Point, South Africa. Annals of the South African Museum, 73, 93-104.

Griffiths, H. J., Whittle, R. J., Roberts, S. J., Belchier, M., \& Linse, K. (2013). Antarctic Crabs: Invasion or Endurance? PLoS ONE, 8(7): e66981.

Guerra-García, J. M. (2001). Habitat use of Caprellidea (Crustacea, Amphipoda) from Ceuta, North Africa. Ophelia, 55, 27-38.

Guerra-García J. M. (2003). The caprellidean Amphipoda from the subantartic islands of New Zealand and Australia with the description of a new genus and two new species. Scientia Marina, 67, 177-194.

Guerra-García, J. M., \& Thiel, M. (2001). La fauna 
de caprélidos (Crustacea: Amphipoda: Caprellidea) de la costa de Coquimbo, centronorte de Chile, con una clave taxonómica para la identificación de las especies. Revista Chilena de Historia Natural, 74, 873-883.

Laubitz, D. R. (1970). Studies on the Caprellidae (Crustacea, Amphipoda) of the American North Pacific. Publications in Biological Oceanography, National Museum of Natural Sciences, 1, 1-89.

Laubitz, D. R., \& Mills, E. L. (1972). Deep-sea Amphipoda from the western North Atlantic Ocean. Caprellidea. Canadian Journal of Zoology, 50, 371-383.

Lewbel, G. S. (1978). Sexual dimorphism and intraspecific aggression and their relationship to sex ratios in Caprella gorgonia Laubitz \& Lewbel (Crustacea:Amphipoda: Caprellidae). Journal of Experimental Marine Biology and Ecology, 33, 133-151.

Lovrich, G., \& Tapella, F. (2014). Southern king crabs. En B. G. Stevens (Ed.), King crabs of the world: biology and fisheries management (608 p.). Boca Ratón: CRC Press, Taylor \& Francis Group.

Martin, D., \& Britayev, T. A. (1998). Symbiotic Polychaetes: Review of known species. Oceanogr. Marine Biology Annual Review, 36, 217-340.

Martin, J. W., \& Pettit, G. (1998). Caprella bathytatos new species (Crustacea, Amphipoda, Caprellidae), from the mouthparts of the crab Macroregonia macrochira Sakai (Brachyura, Majidae) in the vicinity of deep-sea hydrothermal vents off British Columbia. Bulletin of Marine Science, 63, 189-198.

Mayer, P. (1890). Die Caprelliden des Golfes von Neapel und der angrenzenden MeeresAbschnitte. Nachtrag zur Monographie derselben. Fauna Flora Golf. Neapel, 17, 1-157, pls, 1-7.

Mayer, P. (1903). Die Caprellidae der SibogaExpedition, Siboga-Expeditie, 34, 1-160.

McCain, J. C. (1968). The Caprellidae (Crustacea: Amphipoda) of the western North Atlantic. United State National Museum Bulletin, 278, 1-147.

McCain, J. C., \& Steinberg, J. E. (1970).
Amphipoda I. Caprellidea I. Fam. Caprellidae. Crustacean Catalogue, 2, 1-78.

Rozbaczylo, N., \& Cañete, J. I. (1993). A new species of scale-worm, Harmothoe commensalis (Polychaeta: Polynoidae), from mantle cavities of two Chilean clams. Proceedings of the Biological Society of Washington, 106, 666-672.

Schellenberg, A. (1926). Die Caprelliden und Neoxenodice caprellinoides n. g. n. sp. der Deutschen Sudpolar Expedition 19011903. Deutsch. Südpol. Exped, 18(Zool. 10), 465-476.

Schellenberg, A. (1931). Gammariden und Caprelliden des Magellangebietes, Südgeorgiens und der Westantarktis. Further Zoological Results of the Swedish Antarctic Expedition 1901-1903, 2, $1-290$.

Serejo, C. S. (1998). Gammaridean and caprellidean fauna (Crustacea) associated with the sponge Dysidea fragilis Johnston at Arraial do Cabo, Rio de Janeiro, Brazil. Bulletin of Marine Science, 63, 363-385.

Servicio Nacional de Pesca (SERNAPESCA) (2015). Anuario estadístico de pesca (www. sernapesca.cl). Visitado en diciembre, 2016.

Sittrop, D. J. P., \& Serejo, C. S. (2006). A new record of Caprella ungulina Mayer, 1903 (Crustacea: Amphipoda) from Brazilian deep water. Nauplius, 14, 43-48.

Smith, C. R., Grange, L. J., Honig, D. L., Naudts, L., Huber, B., Guidi, L., \& Domack, E. (2011). A large population of king crabs in Palmer Deep on the west Antarctic Peninsula shelf and potential invasive impacts. Proceedings of the Royal Society B-Biol Sci, 279, 1017-1026. Stentiford, G. D., Bateman, K. S., Feist, S. W., Oyarzún, S., Uribe, J. C., Palacios, M., \& Stone, D. M. (2014). Areospora rohanae n.gen. n.sp. (Microsporidia; Areosporiidae n. fam.) elicits multi-nucleate giant-cell formation in southern king crab (Lithodes santolla). Journal of Invertebrate Pathology, 118, 1-11.

Stephensen, K. (1944). Crustacea Malacostraca VIII (Amphipod IV). The Danish Ingolf Expedition, 3(13), 1-51. 
Stevens, B. G. (2014). King crabs of the world: biology and fisheries management. Boca Ratón: CRC Press, Taylor \& Francis Group.

Takeuchi, I., \& Hirano, R. (1995). Clinging behavior of the epifaunal caprellids (Amphipoda) inhabiting the Sargassum zone on the Pacific coast of Japan, with its evolutionary implications. Journal of Crustacean Biology, 15, 481-492.

Takeuchi, I., Takeda, M., \& Takeshita, K. (1989). Redescription of the Bathyal Caprellid, Caprella ungulina Mayer, 1902 (Crustacea, Amphipoda) from the North Pacific. Bulletin of the National Science Museum of Tokyo, Ser. A, 15, 19-28.

Takeuchi, I., Yamakawa, H., \& Fujiwara, M. (1990). The Caprellidea (Crustacea: Amphipoda) inhabiting the green alga Cladophora wrightiana: the influence of wave exposure on the species composition. La mer, 28, 139145.

Thiel, M., Guerra-García, J. M., Lancellotti, D. A., \& Vásquez, N. (2003). The distribution of littoral caprellids (Crustacea: Amphipoda: Caprellidea) along the Pacific coast of continental Chile. Revista Chilena de Historia Natural, 76, 297-312.

Thurston, M. H., \& Bett, B. J. (1993). Eyelessness in marine gammaridean Amphipoda (Crustacea): geographical, bathymetric and taxonomic considerations. Journal of Natural History, 27, 861-881.

Vader, W. (1978). Associations between amphipods and echinoderms. Astarte, 11, 123-134.

Vader, W., \& Tandberg, A. H. S. (2015). Amphipods as associates of other Crustacea: a survey. Journal of Crustacean Biology, 35, 522532.

Vassilenko, S, V. (1972). New species of genus Caprella (Amphipoda, Caprellidae) from the Sea of Okhotsk and the north-west Pacific. Trudy Zool. Institute, Acad. Sci. USSR 52, 223-236.

Vassilenko, S. V. (1974). Caprellids of the sea of USSR and adjacent waters. Opredel po Fn. USSR, 107, 1-287.

Vinuesa, J. H., Lourich, G. A., Tapella, F., \& Pettovello, A. (2015). Capítulo 2: Crustáceos de interés comercial. En $\mathrm{H}$. E. Zaixso \& A. L. Boraso (Eds.), La Zona Costera Patagónica Argentina. Volumen I. Recursos Biológicos Bentónicos (pp, 61-117). Comodoro Rivadavia: Editorial Universitaria de la Patagonia.

Wicksten, M. K. (1982). Crustaceans from baited traps and gill net off southern California. California Fish and Game, 68, 244-248. 
